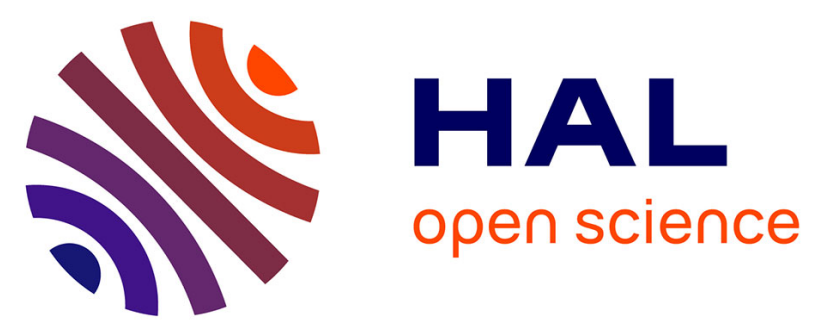

\title{
Metal-Free Addition of Benzyl Halides to Aldehydes Using Super Electron Donors: Access to 3,4-Dihydroisocoumarins and 1,2-Diarylethanols
}

Cédric Spitz, Mélanie Matteudi, Guillaume Tintori, Julie Broggi, Thierry Terme, Patrice Vanelle

\section{To cite this version:}

Cédric Spitz, Mélanie Matteudi, Guillaume Tintori, Julie Broggi, Thierry Terme, et al.. MetalFree Addition of Benzyl Halides to Aldehydes Using Super Electron Donors: Access to 3,4Dihydroisocoumarins and 1,2-Diarylethanols. Journal of Organic Chemistry, 2020, 85 (23), pp.1573615742. 10.1021/acs.joc.0c02374 . hal-03254096

\section{HAL Id: hal-03254096 \\ https://hal-amu.archives-ouvertes.fr/hal-03254096}

Submitted on 8 Jun 2021

HAL is a multi-disciplinary open access archive for the deposit and dissemination of scientific research documents, whether they are published or not. The documents may come from teaching and research institutions in France or abroad, or from public or private research centers.
L'archive ouverte pluridisciplinaire HAL, est destinée au dépôt et à la diffusion de documents scientifiques de niveau recherche, publiés ou non, émanant des établissements d'enseignement et de recherche français ou étrangers, des laboratoires publics ou privés. 


\title{
Metal-Free Addition of Benzyl Halides to Aldehydes Using Super Electron Donors: Access to 3,4-Dihydroisocoumarins and 1,2- Diarylethanols.
}

\author{
Cédric Spitz*, Mélanie Matteudi, Guillaume Tintori, Julie Broggi, Thierry Terme, and Patrice Vanelle*
}

Aix Marseille Univ, CNRS, ICR UMR CNRS 7273, Equipe Pharmaco-Chimie Radicalaire, Faculté de Pharmacie, 27

Boulevard Jean Moulin - CS 30064, 13385 Marseille Cedex 05, France

cedric.spitz@univ-amu.fr, patrice.vanelle@univ-amu.fr

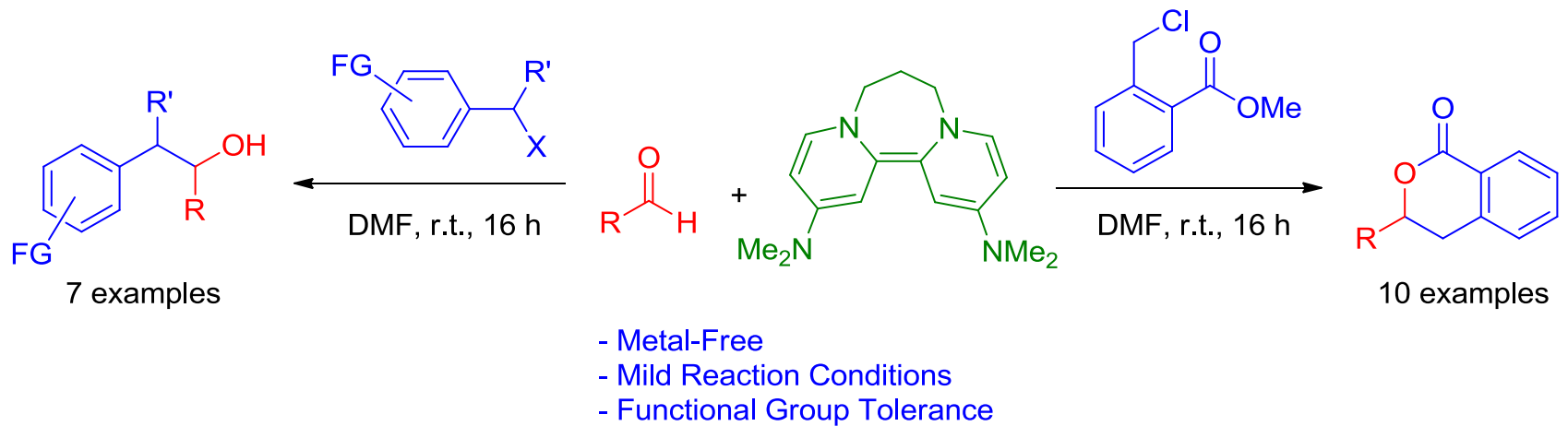

\begin{abstract}
We report here the intermolecular metal-free addition reaction of functionalized benzyl halides to aldehydes using a super electron donor (SED). The metal-free and mild conditions allowed the formation of 3,4-dihydroisocoumarins and 1,2diarylethanols with unprecedented functional group tolerance.
\end{abstract}

The 3,4-dihydroisocoumarin scaffold is found in several natural products and is known for its antimalarial, antituberculous, antifungal and antiproliferative properties. The spatially related 1,2-diarylethanols are also structural motifs in natural products and biologically important molecules. ${ }^{2}$ Thus, numerous strategies have been investigated to allow the construction of these skeletons. ${ }^{3}$ Recently, the synthesis of a 3,4-dihydroisocoumarin was allowed by a metal-free intramolecular direct oxidative lactonization of carboxylic acids using iodine and a photoredox catalyst. ${ }^{4}$ However, most of methodologies synthesizing 3,4dihydroisocoumarins or 1,2-diarylethanols still need the use of strong bases or organometallics. One major limitation of these bases and these reactive organometallics of $\mathrm{Mg}, \mathrm{Li}, \mathrm{Zn}, \mathrm{Mn}$ and $\mathrm{Ti}$ is their poor functional group tolerance.

Tetrakis(dimethylamino)ethylene (TDAE) is an organic reducing agent, which reacts with halogenated derivatives to generate a carbanion under mild conditions. ${ }^{5}$ It was for a long period the most used organic electron donor (OED) thanks to its commercial availability. A comprehensive study of the reducing reactivity of TDAE revealed that a strong electronwithdrawing group (e.g. $\mathrm{NO}_{2}$ or $\mathrm{CN}$ ) is required on the benzylic substrate to reductively cleave the carbon-halogen bond. This constitutes the major drawback of this strategy. Since 2005, Murphy and co-workers developed new reducing agents with neutral organic structures and exceptionally negative redox potentials ${ }^{6}$ (Figure 1). More recently, Dyker and co-workers, with a tetra(iminophosphorano)-substituted bispyridinylidene, ${ }^{7}$ and Wenger and co-workers, using photoexcitation of TDAE ${ }^{8}$ improved even more the reduction potential of organic electron donors.

These "super electron donors" (SEDs) are capable of spontaneous Single Electron Transfer (SET) and Double Electron Transfer (DET) affording the formation of radicals or anions from non-activated benzyl halides, aryl halides, sulfones, triflate esters and triflamides. ${ }^{9}$ They operate under mild conditions and are selective and tolerant to functional groups (nitro, carbonyl, ester, cyano ...) compared to metallic reducers. Furthermore, the use of expensive metal derivatives that cause environmental and economic problems can be avoided. This approach resulted in excellent reducing agents that could be used for organic transformations. However, despite this important gain in reduction power, the reactivity of SEDs has been scarcely studied for carbon-carbon bondforming reactions. SEDs have been recently used as initiators of base-induced homolytic aromatic substitution (BHAS) for the carbon-carbon coupling of haloarenes and arenes. ${ }^{10}$ However, in the role of reducer for the generation of key active species for addition reactions to electrophiles, only intramolecular reactions have been hitherto described. ${ }^{6,11}$

Figure 1. Example of Organic Electron Donors
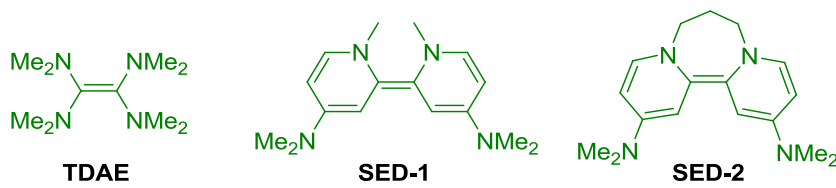
Our aim was to determine if these SEDs could allow intermolecular addition reactions of diversely functionalized benzyl halides to aldehydes under metal-free and mild conditions.

The reaction between methyl 2-(chloromethyl)benzoate $\mathbf{1}$ and benzaldehyde 2a in presence of an OED was selected as a benchmark reaction (Table 1). As expected, the commercially available TDAE was not powerful enough to reduce the benzyl chloride derivative 1 (Table 1, entry 1 ). The low reactivity of methyl 2-(chloromethyl)benzoate 1 to TDAE compared to nitro or cyano benzyl halides can be explained by the lower electron-withdrawing effect of ester compared to $\mathrm{NO}_{2}$ or $\mathrm{CN}$. This renders the methyl 2-(chloromethyl)benzoate $\mathbf{1}$ more difficult to reduce compared to nitro or cyano benzyl halides. Interestingly, the more powerful SED-1, in situ generated from pyridinium precursor salt P-1 and a strong base like KHMDS, allowed the reduction of the methyl 2-(chloromethyl)benzoate 1 in DMF. The nucleophilic addition of the benzylic anion to benzaldehyde $\mathbf{2 a}$, followed by intramolecular cyclisation of the alcoholate on the ester gave the 3,4-dihydroisocoumarin 3a in $39 \%$ yield (entry 2 ). This nucleophilic addition on an aldehyde moiety of the reduced benzylic species confirms the generation of an anion by DET rather than a benzyl radical. Indeed, radical addition to carbonyls is a reversible process due to the formation of a thermodynamically unfavorable alkoxy radical. ${ }^{12}$ We next investigated the effect of the solvent and only traces of product were obtained in acetonitrile and tetrahydrofuran (entries 3-4). Using more equivalents (entry 5) or less equivalents (entry 6) of P-1 and KHMDS were detrimental to the reaction. Using methyl 2-(chloromethyl)benzoate $\mathbf{1}$ in excess compared to benzaldehyde $\mathbf{2 a}$ was not beneficial (entry 7). The amount of solvent slightly influenced the reaction (entry $2 v s$ entry 8). Changing the temperature of the reaction (entries 9-10) showed that room temperature advantageously remains the best choice. No significant influence of the base was observed using either KHMDS or $\mathrm{NaH}$ (entry 8 vs entry 11). SED-2, in situ generated from pyridinium precursor salt $\mathbf{P}$ 2 and KHMDS, was also tested in the reaction and a slightly better yield was obtained (45\%, entry 12). Finally, directly using the isolated SED-2 gave an improved yield of 64\% (entry 13).

\section{Table 1. Optimization of Reaction Conditions}
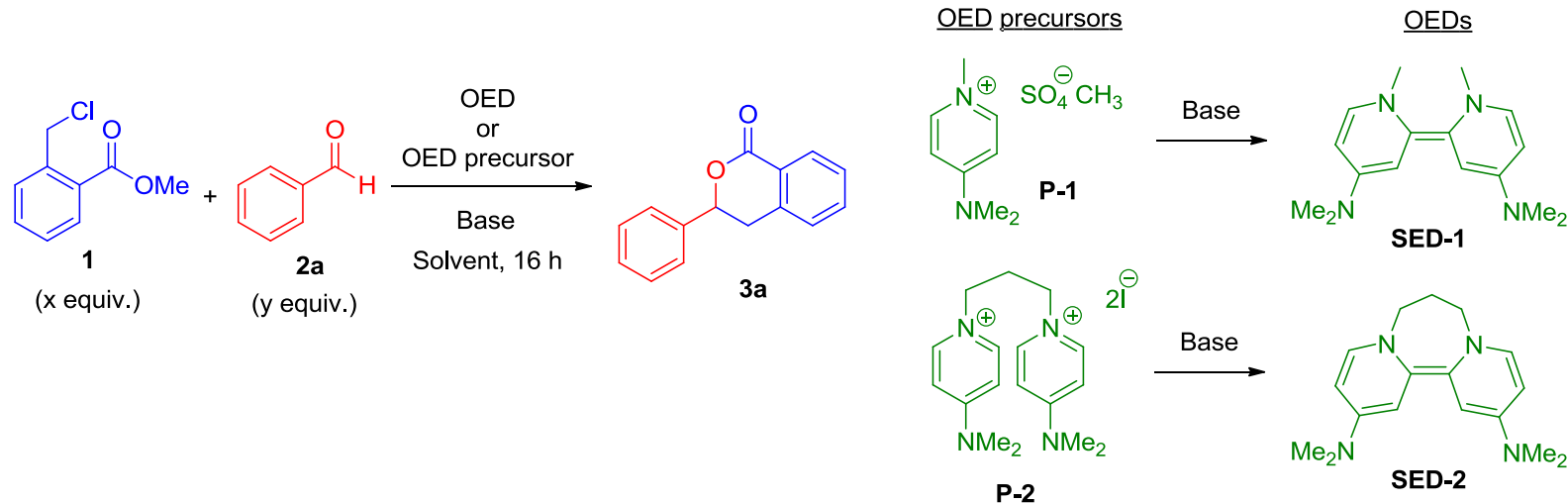

\begin{tabular}{|c|c|c|c|c|c|c|c|}
\hline Entry & $\mathrm{x}$ equiv. & y equiv. & $\begin{array}{c}\text { OED } \\
\text { or } \\
\text { OED precursor } \\
\text { (equiv.) }\end{array}$ & Base (equiv.) & Solvent [M] & $\mathrm{T}\left({ }^{\circ} \mathrm{C}\right)$ & Yield (\%) \\
\hline 1 & 1 & 3 & TDAE (3) & - & DMF [0.04] & rt & 0 \\
\hline 2 & 1 & 3 & P-1 (3) & KHMDS (3) & $\mathrm{DMF}[0.04]$ & $\mathrm{rt}$ & 39 \\
\hline 3 & 1 & 3 & P-1 (3) & KHMDS (3) & $\mathrm{MeCN}[0.04]$ & $\mathrm{rt}$ & traces \\
\hline 4 & 1 & 3 & P-1 (3) & KHMDS (3) & THF [0.04] & $\mathrm{rt}$ & traces \\
\hline 5 & 1 & 3 & P-1 (10) & KHMDS (10) & DMF [0.04] & $\mathrm{rt}$ & 20 \\
\hline 6 & 1 & 3 & P-1 (2) & KHMDS (2) & DMF [0.04] & $\mathrm{rt}$ & 11 \\
\hline 7 & 2 & 1 & P-1 (4) & KHMDS (4) & DMF [0.04] & $\mathrm{rt}$ & 12 \\
\hline 8 & 1 & 3 & P-1 (3) & KHMDS (3) & DMF [0.2] & $\mathrm{rt}$ & 42 \\
\hline 9 & 1 & 3 & P-1 (3) & KHMDS (3) & DMF [0.2] & 0 & 18 \\
\hline 10 & 1 & 3 & P-1 (3) & KHMDS (3) & DMF [0.2] & $40^{\circ} \mathrm{C}$ & traces \\
\hline 11 & 1 & 3 & P-1 (3) & $\mathrm{NaH}(3)$ & DMF [0.2] & $\mathrm{rt}$ & 40 \\
\hline 12 & 1 & 3 & P-2 (1.5) & KHMDS (3) & DMF [0.2] & $\mathrm{rt}$ & 45 \\
\hline 13 & 1 & 3 & SED-2 (1.5) & - & DMF [0.2] & $\mathrm{rt}$ & 64 \\
\hline
\end{tabular}


With the optimized conditions in hand, we next investigated the generality and scope of this reaction with a series of aldehydes $\mathbf{2 a - j}$ (Scheme 1). To our delight, moderate to good yields of 3,4-dihydroisocoumarins were obtained with both electron-poor (3b-e) and electron-rich substituents (3f). The position of the substituent seemed to have little effect on the reaction. The use of cinnamaldehyde allowed the formation of the 3,4-dihydroisocoumarin $\mathbf{3 g}$ resulting from a regioselective 1,2-addition, no 1,4-addition was observed. Interestingly, the reaction conditions are tolerant with the formation of isocoumarins $\mathbf{3 h}$ and $\mathbf{3 i}$ respectively from heteroaromatic aldehydes $\mathbf{2 h}$ and $\mathbf{2 i}$. Finally, a disubstituted aldehyde $\mathbf{2 j}$ allowed the formation of the 3,4-dihydroisocoumarin $\mathbf{3 j}$ with a $55 \%$ yield. Thus, we showed that our reaction conditions are compatible with an ester moiety on the benzyl chloride derivative and diversely functionalized aldehydes. The intermolecular addition of the metal-free generated benzylic anion, followed by a cyclisation, allowed the synthesis of ten 3,4-dihydroisocoumarins in one-step.

\section{Scheme 1. Scope With Diverse Aldehyde Derivatives. ${ }^{\text {a }}$}
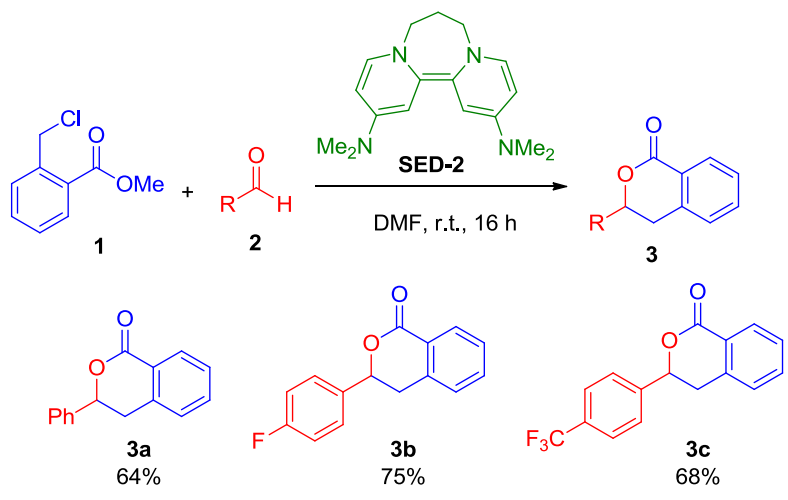

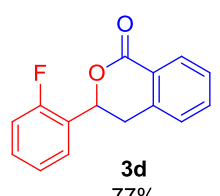

$3 \mathbf{d}$
$77 \%$

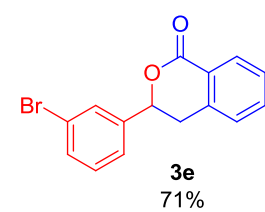

$3 \mathbf{e}$
$71 \%$
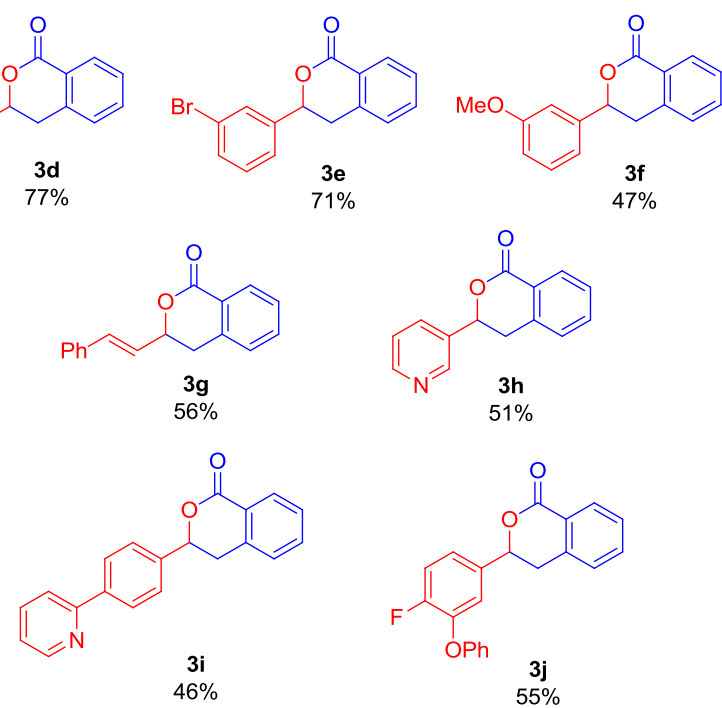

\footnotetext{
${ }^{a}$ Reaction Conditions: 1 (0.2 mmol), 2 (0.6 mmol) and SED-2 (0.3 $\mathrm{mmol})$ in DMF $(1 \mathrm{~mL})$ were stirred at $\mathrm{rt}$ for $16 \mathrm{~h}$ under an inert atmosphere.
}

To further outline the utility of this method, we next examined the scope of the reaction with diversely functionalized benzyl halide derivatives (Scheme 2). Shifting the ester moiety from ortho to para position allowed the synthesis of alcohol 5a (52\% yield) from benzaldehyde $\mathbf{2 a}$ and alcohol 5b (64\% yield) from 4-fluorobenzaldehyde $\mathbf{2 b}$. Contrary to our previous observations with methyl 2(chloromethyl)benzoate $\mathbf{1}$, the intramolecular cyclization of the alcoholate on the ester was not observed with methyl 4(bromomethyl)benzoate because, as expected, the alcoholate was too far to attack on the ester. To our delight, an amide moiety was well tolerated and allowed the synthesis of alcohol $\mathbf{5 c}$ in $42 \%$ yield. To the best of our knowledge, the addition of a benzyl halide derivative, possessing an amide function, on an aldehyde was never described. Interestingly, the super electron donor SED-2 was very selective as it only reduced the chlorine atom of the 4-vinylbenzyl chloride to give alcohol $\mathbf{5 d}$ in $43 \%$ yield. Addition on the double bond was not observed so it confirmed that DET was rapid and that the benzyl radical lifetime is short. The challenging 2-chlorobenzyl chloride was also compatible with our metal-free reaction conditions as the benzylic chlorine atom was preferentially reduced to allow the synthesis of alcohol 5e in $30 \%$ yield. The even more challenging 2-iodobenzyl bromide, as it was described that SDEs can reduce iodoaryl derivatives, ${ }^{6 a}$ allowed the alcohol $\mathbf{5 f}$ in a modest $25 \%$ yield. Interestingly, a secondary benzylic halide was compatible with our strategy and alcohol $\mathbf{5 g}$ was obtained with a $52 \%$ yield. Here, we showed that diversely functionalized benzyl halide reagents can be selectively reduced by a super electron donor to form the corresponding benzylic carbanion under mild conditions. This anion can then be added to aldehydes to give the corresponding 1,2diarylethanols.

Scheme 2. Scope With Diverse Benzyl Halide Derivatives. ${ }^{\text {a }}$

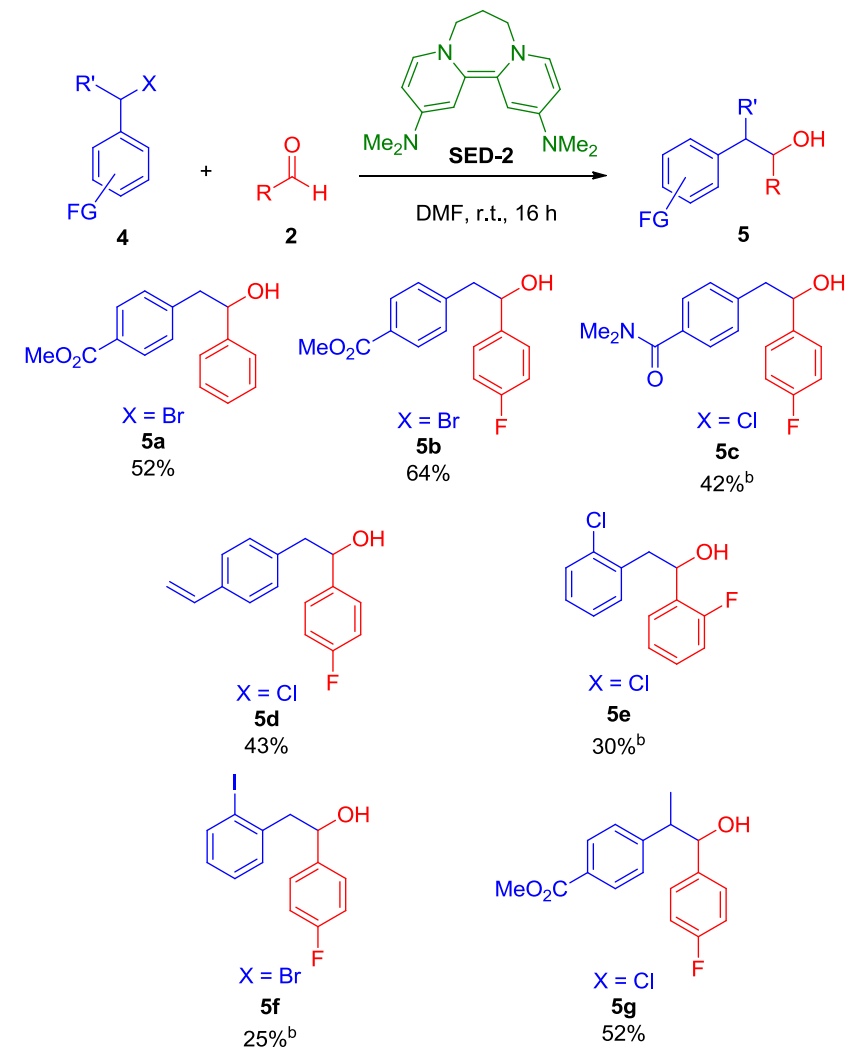

${ }^{a}$ Reaction Conditions: $4(0.2 \mathrm{mmol}), 2(0.6 \mathrm{mmol})$ and SED-2 $(0.3$ $\mathrm{mmol})$ in DMF $(1 \mathrm{~mL})$ were stirred at $\mathrm{rt}$ for $16 \mathrm{~h}$ under an inert atmosphere. ${ }^{\mathrm{b}} 10$ equivalents of aldehyde were used. 
To support a two consecutive electron transfer mechanism (Scheme 3), the reaction between methyl 2(chloromethyl)benzoate 1, 4-fluorobenzaldehyde and SED-2 was carried out in the presence of TEMPO. The isocoumarin 3b was obtained in only $17 \%$ yield which confirms the intermediacy of a benzyl radical. Furthermore, the second electron transfer to form the anion was supposed to be fast as no complete inhibition was observed with TEMPO and no addition on the double bond was observed during the synthesis of alcohol $\mathbf{5 d}$.

\section{Scheme 3. Proposed DET Reaction Mechanism}

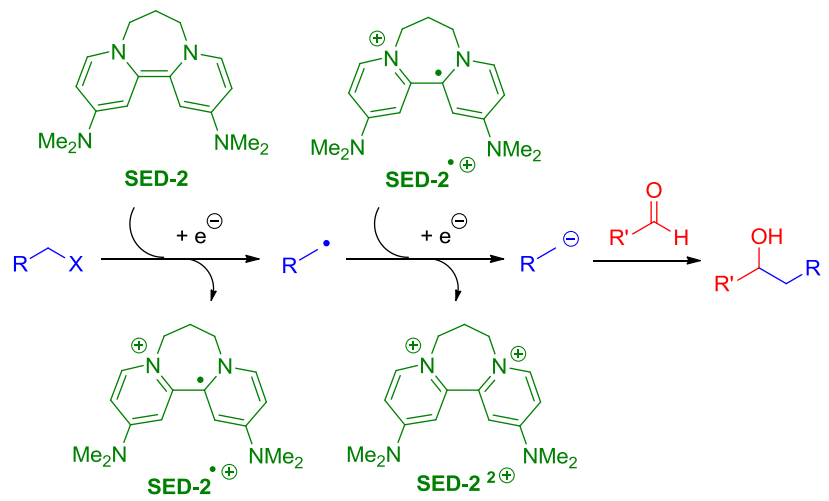

In conclusion, the intermolecular addition of functionalized benzyl halides to aldehydes using a super electron donor SED2 is documented. Thus, a range of 3,4-dihydroisocoumarins and 1,2-diarylethanols was obtained with unprecedented functional group tolerance using metal-free and mild conditions.

\section{EXPERIMENTAL SECTION}

General Information. All reactions were carried out under an argon atmosphere using an oven-dried glassware. All solvents and chemicals were used as purchased without further purification. Melting points were determined on a Büchi melting point B-540 apparatus and are uncorrected. HRMS analyses were performed on a Synapt G2 HDMS (Waters) with a TOF mass analyzer type at the spectropole of Aix-Marseille University. Both ${ }^{1} \mathrm{H}$ - and ${ }^{13} \mathrm{C}-\mathrm{NMR}$ spectra were determined on a Bruker Avance NEO $400 \mathrm{MHz}$ Nanobay spectrometer and on a Bruker AC 250 spectrometer at the Service de RMN de la Faculté de Pharmacie de Marseille of the AixMarseille University. The ${ }^{1} \mathrm{H}$ and the ${ }^{13} \mathrm{C}$ chemical shifts are reported from $\mathrm{CDCl}_{3}$ peaks: ${ }^{1} \mathrm{H}(7.26 \mathrm{ppm})$ and ${ }^{13} \mathrm{C} \quad(77.16 \mathrm{ppm})$. Multiplicities are represented by the following notations: s, singlet; $d$, doublet; t, triplet; q, quartet; $\mathrm{m}$, a more complex multiplet or overlapping multiplets. The following adsorbents were used for column chromatography: Silica gel 60 (particle size $0.063-0.200 \mathrm{~mm}$, 70-230 mesh ASTM). TLC was performed on $5 \times 10 \mathrm{~cm}$ aluminum plates coated with silica gel 60 F254 in an appropriate solvent.

Preparation of P-1. To a solution of DMAP (1.22 g, $10 \mathrm{mmol})$ in toluene $(10 \mathrm{~mL})$, dimethylsulfate $(0.95 \mathrm{~mL}, 10 \mathrm{mmol})$ was added dropwise and the reaction mixture was stirred at $100{ }^{\circ} \mathrm{C}$ for $3 \mathrm{~h}$. After being cooled, the product was filtered, washed with toluene $(20 \mathrm{~mL})$, and dried in vacuo to give a white solid $(2.21 \mathrm{~g}, 89 \%)$. Mp 242-244 ${ }^{\circ} \mathrm{C}$; ${ }^{1} \mathrm{H}$ NMR $\left(400 \mathrm{MHz}, \mathrm{CDCl}_{3}\right) \delta 8.21(\mathrm{~d}, J=7.8 \mathrm{~Hz}, 2 \mathrm{H}), 6.90(\mathrm{~d}, J$ $=7.8 \mathrm{~Hz}, 2 \mathrm{H}), 4.01(\mathrm{~s}, 3 \mathrm{H}), 3.68(\mathrm{~s}, 3 \mathrm{H}), 3.21(\mathrm{~s}, 6 \mathrm{H}) ;{ }^{13} \mathrm{C}\left\{{ }^{1} \mathrm{H}\right\} \mathrm{NMR}$ $\left(101 \mathrm{MHz}, \mathrm{CDCl}_{3}\right) \delta 156.2,143.3,108.2,54.6,45.0,40.3$; HRMS (ESI) : $\mathrm{m} / z\left[2 \mathrm{C}^{+}+\mathrm{A}^{-}\right]^{+}$calcd for $\left[\mathrm{C}_{17} \mathrm{H}_{29} \mathrm{~N}_{4} \mathrm{O}_{4} \mathrm{~S}\right]^{+}: 385.1904$; found : 385.1901

Preparation of SED-2. The procedure, previously reported for the synthesis of SED-2, ${ }^{11}$ was slightly modified: in the glovebox, sodium hydride ( $1.1 \mathrm{~g}, 46 \mathrm{mmol}, 5$ equiv.) was added to a solution of the precursor bis-pyridinium salt ${ }^{11}$ in extra dry $N, N$-dimethylformamide $(20 \mathrm{~mL})$. After $2 \mathrm{~h}$ stirring at room temperature, the reaction mixture was filtrated to remove excess sodium hydride. The grey residue was washed with diethyl ether and solvents were removed under vacuum. Diethyl ether was then added in several fractions to re-dissolve the dark purple bispyridinylidene SED-2. The mixture was filtrated to remove excess sodium iodide. Evaporation of the filtrate afforded SED-2 as a well-defined dark purple solid (2.42 g, 92\%). SED-2 was stable under inert atmosphere and stored in a glovebox. Spectral data match those previously reported. ${ }^{11}$

General Procedures for the Synthesis of isocoumarins 3a-j or alcohols 5a-g.

General Procedure A when the benzyl halide derivative is liquid: in a glove-box, to SED-2 (85 mg, $0.3 \mathrm{mmol}, 1.5$ equiv.) was added dry DMF (1 mL). The previous solution was put out from the glove-box and the aldehyde $(0.6 \mathrm{mmol}, 3$ equiv.) was added followed by the benzyl halide ( $0.2 \mathrm{mmol}, 1$ equiv.) under nitrogen atmosphere. The reaction mixture was stirred at room temperature for $16 \mathrm{~h}$. Water was added $(5 \mathrm{~mL})$ and a $1 \mathrm{M}$ solution of $\mathrm{HCl}$ was added until $\mathrm{pH}=1$. The aqueous layer was extracted with dichloromethane $(3 \times 10 \mathrm{~mL})$. The combined organic layer was dried over $\mathrm{Na}_{2} \mathrm{SO}_{4}$. Evaporation of the solvent furnished the crude product. Purification by silica gel chromatography afforded pure isocoumarins $\mathbf{3 a}-\mathbf{j}$ or alcohols $\mathbf{5 d - e}$.

General Procedure B when the benzyl halide derivative is solid: in a glove-box, to SED-2 (85 mg, $0.3 \mathrm{mmol}, 1.5$ equiv.) was added dry DMF $(0.5 \mathrm{~mL})$ and $0.5 \mathrm{~mL}$ of dry DMF was put in another vial. The solid benzyl halide $(0.2 \mathrm{mmol}, 1$ equiv. $)$ was weight in a Schlenk and solubilized with the previous $0.5 \mathrm{~mL}$ of dry DMF under nitrogen atmosphere. The vial containing SED-2 in DMF was put out from the glove-box and the aldehyde ( $0.6 \mathrm{mmol}, 3$ equiv.) was added followed by the benzyl halide solution. The reaction mixture was stirred at room temperature for $16 \mathrm{~h}$. Water was added $(5 \mathrm{~mL})$ and a $1 \mathrm{M}$ solution of $\mathrm{HCl}$ was added until $\mathrm{pH}=1$. The aqueous layer was extracted with dichloromethane $(3 \times 10 \mathrm{~mL})$. The combined organic layer was dried over $\mathrm{Na}_{2} \mathrm{SO}_{4}$. Evaporation of the solvent furnished the crude product. Purification by silica gel chromatography afforded pure alcohols $\mathbf{5 a}-\mathbf{c}$ and $\mathbf{5 f}-\mathbf{g}$.

3-Phenyl-3,4-dihydroisocoumarin (3a). Following the general procedure A with 2-(chloromethyl)benzoate and benzaldehyde, 3a was purified by $\mathrm{PE} / \mathrm{EtOAc}(90 / 10)$. $28.8 \mathrm{mg}$, yield: $64 \%$; white solid; mp 87-89 ${ }^{\circ} \mathrm{C} ;{ }^{1} \mathrm{H} \mathrm{NMR}\left(250 \mathrm{MHz}, \mathrm{CDCl}_{3}\right) \delta 8.16(\mathrm{~d}, J=7.5 \mathrm{~Hz}, 1 \mathrm{H})$, $7.61-7.31(\mathrm{~m}, 8 \mathrm{H}), 5.57(\mathrm{dd}, J=11.9,3.2 \mathrm{~Hz}, 1 \mathrm{H}), 3.36(\mathrm{dd}, J=16.4$, $12.0 \mathrm{~Hz}, 1 \mathrm{H}), 3.14(\mathrm{dd}, J=16.5,3.2 \mathrm{~Hz}, 1 \mathrm{H})$. Spectral data match those previously reported. ${ }^{13}$

3-(4-fluorophenyl)-3,4-dihydroisocoumarin (3b). Following the general procedure $A$ with 2-(chloromethyl)benzoate and 4fluorobenzaldehyde, 3b was purified by PE/EtOAc (90/10). $36.2 \mathrm{mg}$, yield: 75\%; pale-yellow solid; mp $84-86^{\circ} \mathrm{C}$; ${ }^{1} \mathrm{H}$ NMR $(250 \mathrm{MHz}$, $\left.\mathrm{CDCl}_{3}\right) \delta 8.15(\mathrm{dd}, J=7.8,1.4 \mathrm{~Hz}, 1 \mathrm{H}), 7.58(\mathrm{td}, J=7.5,1.4 \mathrm{~Hz}, 1 \mathrm{H})$, $7.52-7.39(\mathrm{~m}, 3 \mathrm{H}), 7.29(\mathrm{~d}, J=7.6 \mathrm{~Hz}, 1 \mathrm{H}), 7.18-7.05(\mathrm{~m}, 2 \mathrm{H}), 5.54$ $(\mathrm{dd}, J=12.1,3.2 \mathrm{~Hz}, 1 \mathrm{H}), 3.32(\mathrm{dd}, J=16.5,12.1 \mathrm{~Hz}, 1 \mathrm{H}), 3.11(\mathrm{dd}$, $J=16.4,3.2 \mathrm{~Hz}, 1 \mathrm{H})$ Spectral data match those previously reported. ${ }^{14}$ 3-(4-(trifluoromethyl)phenyl)-3,4-dihydroisocoumarin Following the general procedure A with 2-(chloromethyl)benzoate and 4-(trifluoromethyl)benzaldehyde, $3 \mathbf{c}$ was purified by $\mathrm{PE} / \mathrm{EtOAc}$ (90/10). $39.7 \mathrm{mg}$, yield: $68 \%$; white solid; mp $106-108^{\circ} \mathrm{C} ;{ }^{1} \mathrm{H}$ NMR $\left(400 \mathrm{MHz}, \mathrm{CDCl}_{3}\right) \delta 8.17(\mathrm{dd}, J=7.8,0.9 \mathrm{~Hz}, 1 \mathrm{H}), 7.69(\mathrm{~d}, J=8.3$ $\mathrm{Hz}, 2 \mathrm{H}), 7.62(\mathrm{~d}, J=8.2 \mathrm{~Hz}, 2 \mathrm{H}), 7.59(\mathrm{dd}, J=7.5,1.3 \mathrm{~Hz}, 1 \mathrm{H}), 7.46$ $(\mathrm{t}, J=7.6 \mathrm{~Hz}, 1 \mathrm{H}), 7.30(\mathrm{~d}, J=7.6 \mathrm{~Hz}, 1 \mathrm{H}), 5.63(\mathrm{dd}, J=11.9,3.3$ $\mathrm{Hz}, 1 \mathrm{H}), 3.31(\mathrm{dd}, J=16.4,11.9 \mathrm{~Hz}, 1 \mathrm{H}), 3.17(\mathrm{dd}, J=16.4,3.3 \mathrm{~Hz}$, $1 \mathrm{H}) ;{ }^{13} \mathrm{C}\left\{{ }^{1} \mathrm{H}\right\}$ NMR $\left(101 \mathrm{MHz}, \mathrm{CDCl}_{3}\right) \delta 165.0,142.6,138.5,134.3$, $131.4\left(\mathrm{C}-\mathrm{F}_{3},{ }^{2} \mathrm{JC}-\mathrm{F}=33.3 \mathrm{~Hz}\right), 131.1\left(\mathrm{C}-\mathrm{F}_{3},{ }^{2} \mathrm{JC}-\mathrm{F}=33.3 \mathrm{~Hz}\right), 130.8$ $\left(\mathrm{C}-\mathrm{F}_{3},{ }^{2} \mathrm{JC}-\mathrm{F}=33.3 \mathrm{~Hz}\right), 130.7,130.5\left(\mathrm{C}-\mathrm{F}_{3},{ }^{2} \mathrm{JC}-\mathrm{F}=33.3 \mathrm{~Hz}\right), 128.3$, $128.1\left(\mathrm{C}-\mathrm{F}_{3},{ }^{1} \mathrm{JC}-\mathrm{F}=272.7 \mathrm{~Hz}\right), 127.5,126.5,125.92\left(\mathrm{C}-\mathrm{F}_{3},{ }^{3} \mathrm{JC}-\mathrm{F}=\right.$ $3.37 \mathrm{~Hz}), 125.89\left(\mathrm{C}-\mathrm{F}_{3},{ }^{3} \mathrm{JC}-\mathrm{F}=3.37 \mathrm{~Hz}\right) 125.85\left(\mathrm{C}-\mathrm{F}_{3},{ }^{3} \mathrm{JC}-\mathrm{F}=3.37\right.$ $\mathrm{Hz}), 125.81\left(\mathrm{C}-\mathrm{F}_{3},{ }^{3} \mathrm{JC}-\mathrm{F}=3.37 \mathrm{~Hz}\right), 125.4\left(\mathrm{C}-\mathrm{F}_{3},{ }^{1} \mathrm{JC}-\mathrm{F}=272.7 \mathrm{~Hz}\right)$, 125.1, $122.7\left(\mathrm{C}-\mathrm{F}_{3},{ }^{1} \mathrm{JC}-\mathrm{F}=272.7 \mathrm{~Hz}\right), 120.0\left(\mathrm{C}-\mathrm{F}_{3},{ }^{1} \mathrm{JC}-\mathrm{F}=272.7\right.$ 
$\mathrm{Hz}$ ), 79.1, 35.7; HRMS (ESI) : $m / z,[\mathrm{M}+\mathrm{H}]^{+}$calcd for $\left[\mathrm{C}_{16} \mathrm{H}_{12} \mathrm{O}_{2} \mathrm{~F}_{3}\right]^{+}$: 293.0784; found : 293.0782 .

3-(2-fluorophenyl)-3,4-dihydroisocoumarin (3d). Following the general procedure $A$ with 2-(chloromethyl)benzoate and 2fluorobenzaldehyde, 3d was purified by PE/EtOAc (90/10). $37.4 \mathrm{mg}$, yield: $77 \%$; pale-yellow solid; mp $85-87^{\circ} \mathrm{C}$; ${ }^{1} \mathrm{H}$ NMR $(250 \mathrm{MHz}$, $\left.\mathrm{CDCl}_{3}\right) \delta 8.16(\mathrm{dd}, J=7.7,1.1 \mathrm{~Hz}, 1 \mathrm{H}), 7.69-7.54(\mathrm{~m}, 2 \mathrm{H}), 7.46(\mathrm{~d}, J$ $=7.6 \mathrm{~Hz}, 1 \mathrm{H}), 7.38-7.32(\mathrm{~m}, 1 \mathrm{H}), 7.29(\mathrm{~d}, J=7.7 \mathrm{~Hz}, 1 \mathrm{H}), 7.25-7.20$ (m, 1H), 7.13-7.05 (m, 1H), $5.87(\mathrm{dd}, J=11.6,3.6 \mathrm{~Hz}, 1 \mathrm{H}), 3.31(\mathrm{dd}$, $J=16.4,11.6 \mathrm{~Hz}, 1 \mathrm{H}), 3.17(\mathrm{dd}, J=16.4,3.6 \mathrm{~Hz}, 1 \mathrm{H}) ;{ }^{13} \mathrm{C}\left\{{ }^{1} \mathrm{H}\right\} \mathrm{NMR}$ $\left(63 \mathrm{MHz}, \mathrm{CDCl}_{3}\right) \delta 165.3,161.2\left(\mathrm{C}-\mathrm{F},{ }^{1} \mathrm{JC}-\mathrm{F}=219.9 \mathrm{~Hz}\right), 157.7(\mathrm{C}-$ $\left.\mathrm{F},{ }^{1} \mathrm{JC}-\mathrm{F}=219.9 \mathrm{~Hz}\right), 139.0,134.1,130.6,130.3\left(\mathrm{C}-\mathrm{F},{ }^{3} \mathrm{JC}-\mathrm{F}=8.2\right.$ $\mathrm{Hz}), 130.2\left(\mathrm{C}-\mathrm{F},{ }^{3} \mathrm{JC}-\mathrm{F}=8.2 \mathrm{~Hz}\right), 128.1,127.73\left(\mathrm{C}-\mathrm{F},{ }^{4} \mathrm{JC}-\mathrm{F}=3.2\right.$ $\mathrm{Hz}), 127.68\left(\mathrm{C}-\mathrm{F},{ }^{4} \mathrm{JC}-\mathrm{F}=3.2 \mathrm{~Hz}\right), 127.5,126.2\left(\mathrm{C}-\mathrm{F},{ }^{2} \mathrm{JC}-\mathrm{F}=12.6\right.$ $\mathrm{Hz}), 126.0\left(\mathrm{C}-\mathrm{F},{ }^{2} \mathrm{JC}-\mathrm{F}=12.6 \mathrm{~Hz}\right), 125.1,124.71\left(\mathrm{C}-\mathrm{F},{ }^{3} \mathrm{JC}-\mathrm{F}=3.5\right.$ $\mathrm{Hz}), 124.65\left(\mathrm{C}-\mathrm{F},{ }^{3} \mathrm{JC}-\mathrm{F}=3.5 \mathrm{~Hz}\right), 115.8\left(\mathrm{C}-\mathrm{F},{ }^{2} \mathrm{JC}-\mathrm{F}=21.4 \mathrm{~Hz}\right)$, $115.5\left(\mathrm{C}-\mathrm{F},{ }^{2} \mathrm{JC}-\mathrm{F}=21.4 \mathrm{~Hz}\right), 74.27\left(\mathrm{C}-\mathrm{F},{ }^{4} \mathrm{JC}-\mathrm{F}=3.4 \mathrm{~Hz}\right), 74.21(\mathrm{C}-$ $\left.\mathrm{F},{ }^{3} \mathrm{JC}-\mathrm{F}=3.4 \mathrm{~Hz}\right), 34.7\left(\mathrm{C}-\mathrm{F},{ }^{4} \mathrm{JC}-\mathrm{F}=1.4 \mathrm{~Hz}\right)$; HRMS (ESI) $: \mathrm{m} / \mathrm{z}$ $[\mathrm{M}+\mathrm{H}]^{+}$calcd for $\left[\mathrm{C}_{15} \mathrm{H}_{12} \mathrm{O}_{2} \mathrm{~F}\right]^{+}: 243.0816$; found : 243.0815 .

3-(3-bromophenyl)-3,4-dihydroisocoumarin (3e). Following the general procedure $A$ with 2-(chloromethyl)benzoate and 3bromobenzaldehyde, 3e was purified by PE/EtOAc (90/10). $42.8 \mathrm{mg}$, yield: $71 \%$; white solid; mp $113-115^{\circ} \mathrm{C} ;{ }^{1} \mathrm{H}$ NMR $\left(250 \mathrm{MHz}, \mathrm{CDCl}_{3}\right)$ $\delta 8.15(\mathrm{~d}, J=7.6 \mathrm{~Hz}, 1 \mathrm{H}), 7.66-7.55(\mathrm{~m}, 2 \mathrm{H}), 7.52-7.39(\mathrm{~m}, 3 \mathrm{H})$, $7.32-7.27(\mathrm{~m}, 2 \mathrm{H}), 5.52(\mathrm{dd}, J=11.8,3.4 \mathrm{~Hz}, 1 \mathrm{H}), 3.30(\mathrm{dd}, J=16.4$, $11.8 \mathrm{~Hz}, 1 \mathrm{H}), 3.13(\mathrm{dd}, J=16.4,3.4 \mathrm{~Hz}, 1 \mathrm{H})$. Spectral data match those previously reported. ${ }^{3 \mathrm{~d}}$

3-(3-methoxyphenyl)-3,4-dihydroisocoumarin (3f). Following the general procedure A with 2-(chloromethyl)benzoate and 3methoxybenzaldehyde, 3 f was purified by PE/EtOAc (90/10). 24.1 mg, yield: $47 \%$; white solid; mp $98-99^{\circ} \mathrm{C}$; ${ }^{1} \mathrm{H}$ NMR $(400 \mathrm{MHz}$, $\left.\mathrm{CDCl}_{3}\right) \delta 8.16(\mathrm{dd}, J=7.8,1.0 \mathrm{~Hz}, 1 \mathrm{H}), 7.57(\mathrm{td}, J=7.5,1.4 \mathrm{~Hz}, 1 \mathrm{H})$, $7.43(\mathrm{t}, J=7.6 \mathrm{~Hz}, 1 \mathrm{H}), 7.32(\mathrm{t}, J=8.1 \mathrm{~Hz}, 1 \mathrm{H}), 7.29(\mathrm{~d}, J=7.6 \mathrm{~Hz}$, $1 \mathrm{H}), 7.05-7.03(\mathrm{~m}, 2 \mathrm{H}), 6.91$ (ddd, $J=8.3,2.5,0.9 \mathrm{~Hz}, 1 \mathrm{H}), 5.54(\mathrm{dd}$ $J=12.0,3.2 \mathrm{~Hz}, 1 \mathrm{H}), 3.84(\mathrm{~s}, 3 \mathrm{H}), 3.34(\mathrm{dd}, J=16.4,12.0 \mathrm{~Hz}, 1 \mathrm{H})$, $3.14(\mathrm{dd}, J=16.4,3.2 \mathrm{~Hz}, 1 \mathrm{H})$. Spectral data match those previously reported. ${ }^{15}$

3-styryl-3,4-dihydroisocoumarin (3g). Following the general procedure A with 2-(chloromethyl)benzoate and cinnamaldehyde, $\mathbf{3 g}$ was purified by PE/EtOAc (90/10). $28.2 \mathrm{mg}$, yield: $56 \%$; white solid; $\mathrm{mp} 73-75^{\circ} \mathrm{C} ;{ }^{1} \mathrm{H}$ NMR $\left(400 \mathrm{MHz}, \mathrm{CDCl}_{3}\right) \delta 8.13(\mathrm{~d}, J=7.6 \mathrm{~Hz}, 1 \mathrm{H})$, $7.56(\mathrm{td}, J=7.5,1.0 \mathrm{~Hz}, 1 \mathrm{H}), 7.46-7.27(\mathrm{~m}, 7 \mathrm{H}), 6.79(\mathrm{~d}, J=16.0$ $\mathrm{Hz}, 1 \mathrm{H}), 6.34(\mathrm{dd}, J=16.0,6.4 \mathrm{~Hz}, 1 \mathrm{H}), 5.21(\mathrm{td}, J=9.4,4.7 \mathrm{~Hz}$, 1H), 3.19 (dd, $J=16.3,10.3 \mathrm{~Hz}, 1 \mathrm{H}), 3.11$ (dd, $J=16.3,4.0 \mathrm{~Hz}, 1 \mathrm{H}$ ). Spectral data match those previously reported. ${ }^{13}$

3-(pyridin-3-yl)-3,4-dihydroisocoumarin (3h). Following the general procedure A with 2-(chloromethyl)benzoate and 3pyridinecarboxaldehyde, $3 \mathbf{h}$ was purified by PE/EtOAc (from 50/50 to 20/80). $22.9 \mathrm{mg}$, yield: $51 \%$; white solid; $\mathrm{mp} 80-82{ }^{\circ} \mathrm{C}$; ${ }^{1} \mathrm{H}$ NMR $\left(250 \mathrm{MHz}, \mathrm{CDCl}_{3}\right) \delta 8.74-8.60(\mathrm{~m}, 2 \mathrm{H}), 8.14(\mathrm{~d}, J=7.7 \mathrm{~Hz}, 1 \mathrm{H})$, $7.87(\mathrm{~d}, J=7.9 \mathrm{~Hz}, 1 \mathrm{H}), 7.65-7.53(\mathrm{~m}, 1 \mathrm{H}), 7.46(\mathrm{~d}, J=7.6 \mathrm{~Hz}, 1 \mathrm{H})$, $7.42-7.34(\mathrm{~m}, 1 \mathrm{H}), 7.30(\mathrm{~d}, J=7.5 \mathrm{~Hz}, 1 \mathrm{H}), 5.61(\mathrm{dd}, J=11.9,3.3$ $\mathrm{Hz}, 1 \mathrm{H}), 3.35(\mathrm{dd}, J=16.3,12.0 \mathrm{~Hz}, 1 \mathrm{H}), 3.16(\mathrm{dd}, J=16.4,3.3 \mathrm{~Hz}$, $1 \mathrm{H}) ;{ }^{13} \mathrm{C}\left\{{ }^{1} \mathrm{H}\right\}$ NMR $\left(63 \mathrm{MHz}, \mathrm{CDCl}_{3}\right) \delta 164.9,150.2,147.8,138.4$, 134.34, 134.29, 134.1, 130.7, 128.3, 127.5, 125.0, 123.8, 77.8, 35.4; HRMS (ESI) : $\mathrm{m} / z[\mathrm{M}+\mathrm{H}]^{+}$calcd for $\left[\mathrm{C}_{14} \mathrm{H}_{12} \mathrm{NO}_{2}\right]^{+}: 226.0863$; found $: 226.0862$.

3-(4-(pyridin-2-yl)phenyl)-3,4-dihydroisocoumarin (3i). Following the general procedure $\mathrm{A}$ with 2-(chloromethyl)benzoate and 4(pyridin-2-yl)benzaldehyde, 3i was purified by $\mathrm{PE} / \mathrm{EtOAc}$ (from 90/10 to 80/20). $27.5 \mathrm{mg}$, yield: $46 \%$; white solid; mp $107-109{ }^{\circ} \mathrm{C} ;{ }^{1} \mathrm{H}$ NMR (400 MHz, $\left.\mathrm{CDCl}_{3}\right) \delta 8.72(\mathrm{~d}, J=4.6 \mathrm{~Hz}, 1 \mathrm{H}), 8.17(\mathrm{~d}, J=8.0$ $\mathrm{Hz}, 1 \mathrm{H}), 8.06(\mathrm{~d}, J=8.4 \mathrm{~Hz}, 2 \mathrm{H}), 7.82-7.75(\mathrm{~m}, 2 \mathrm{H}), 7.62-7.56(\mathrm{~m}$, $3 \mathrm{H}), 7.44(\mathrm{t}, J=7.6 \mathrm{~Hz}, 1 \mathrm{H}), 7.32-7.27(\mathrm{~m}, 2 \mathrm{H}), 5.64(\mathrm{dd}, J=11.8$, $3.2 \mathrm{~Hz}, 1 \mathrm{H}), 3.37(\mathrm{dd}, J=16.4,11.9 \mathrm{~Hz}, 1 \mathrm{H}), 3.19(\mathrm{dd}, J=16.5,3.2$ $\mathrm{Hz}, 1 \mathrm{H}) ;{ }^{13} \mathrm{C}\left\{{ }^{1} \mathrm{H}\right\}$ NMR $\left(101 \mathrm{MHz}, \mathrm{CDCl}_{3}\right) \delta 165.4,156.8,149.7$, 139.6, 139.5, 139.0, 137.2, 134.1, 130.6, 128.1, 127.5, 127.4, 126.6,
125.3, 122.6, 120.9, 79.7, 35.7; HRMS (ESI) : $\mathrm{m} / \mathrm{z}[\mathrm{M}+\mathrm{H}]^{+}$calcd for $\left[\mathrm{C}_{20} \mathrm{H}_{16} \mathrm{NO}_{2}\right]^{+}$: 302.1176; found : 302.1172 .

3-(4-fluoro-3-phenoxyphenyl)-3,4-dihydroisocoumarin

Following the general procedure A with 2-(chloromethyl)benzoate and 4-fluoro-3-phenoxybenzaldehyde, $3 \mathbf{j}$ was purified by $\mathrm{PE} / \mathrm{EtOAc}$ (90/10). $36.6 \mathrm{mg}$, yield: $55 \%$; white solid; $\mathrm{mp} 88-90{ }^{\circ} \mathrm{C}$; ${ }^{1} \mathrm{H}$ NMR $\left(400 \mathrm{MHz}, \mathrm{CDCl}_{3}\right) \delta 8.11(\mathrm{~d}, J=7.7 \mathrm{~Hz}, 1 \mathrm{H}), 7.55(\mathrm{t}, J=7.2 \mathrm{~Hz}$, $1 \mathrm{H}), 7.41(\mathrm{t}, J=7.6 \mathrm{~Hz}, 1 \mathrm{H}), 7.32(\mathrm{t}, J=7.9 \mathrm{~Hz}, 2 \mathrm{H}), 7.28-7.14(\mathrm{~m}$, $4 \mathrm{H}), 7.10(\mathrm{t}, J=7.4 \mathrm{~Hz}, 1 \mathrm{H}), 6.97(\mathrm{~d}, J=8.0 \mathrm{~Hz}, 2 \mathrm{H}), 5.46(\mathrm{dd}, J=$ $12.0,2.9 \mathrm{~Hz}, 1 \mathrm{H}), 3.27$ (dd, $J=16.3,12.1 \mathrm{~Hz}, 1 \mathrm{H}), 3.08$ (dd, $J=16.4$, $3.0 \mathrm{~Hz}, 1 \mathrm{H}) ;{ }^{13} \mathrm{C}\left\{{ }^{1} \mathrm{H}\right\}$ NMR $\left(101 \mathrm{MHz}, \mathrm{CDCl}_{3}\right) \delta 165.1,157.1,155.6$ $\left(\mathrm{C}-\mathrm{F},{ }^{1} \mathrm{JC}-\mathrm{F}=251.5 \mathrm{~Hz}\right), 153.1\left(\mathrm{C}-\mathrm{F},{ }^{1} \mathrm{JC}-\mathrm{F}=251.5 \mathrm{~Hz}\right), 144.2(\mathrm{C}-\mathrm{F}$, $\left.{ }^{2} \mathrm{JC}-\mathrm{F}=11.7 \mathrm{~Hz}\right), 144.1\left(\mathrm{C}-\mathrm{F},{ }^{2} \mathrm{JC}-\mathrm{F}=11.7 \mathrm{~Hz}\right), 138.7,135.60(\mathrm{C}-\mathrm{F}$, $\left.{ }^{3} \mathrm{JC}-\mathrm{F}=3.7 \mathrm{~Hz}\right), 135.57\left(\mathrm{C}-\mathrm{F},{ }^{3} \mathrm{JC}-\mathrm{F}=3.7 \mathrm{~Hz}\right), 134.2,130.6,130.0$, 128.1, 127.5, 125.0, 123.6, $122.7\left(\mathrm{C}-\mathrm{F},{ }^{3} \mathrm{JC}-\mathrm{F}=7.2 \mathrm{~Hz}\right), 122.6(\mathrm{C}-\mathrm{F}$, $\left.{ }^{3} \mathrm{JC}-\mathrm{F}=7.2 \mathrm{~Hz}\right), 119.83\left(\mathrm{C}-\mathrm{F},{ }^{4} \mathrm{JC}-\mathrm{F}=1.2 \mathrm{~Hz}\right), 119.82\left(\mathrm{C}-\mathrm{F},{ }^{4} \mathrm{JC}-\mathrm{F}=\right.$ $1.2 \mathrm{~Hz}), 117.6,117.6$ (C-F, $\left.{ }^{2} \mathrm{JC}-\mathrm{F}=20.1 \mathrm{~Hz}\right), 117.4\left(\mathrm{C}-\mathrm{F},{ }^{2} \mathrm{JC}-\mathrm{F}=\right.$ $20.1 \mathrm{~Hz}), 79.1,35.6$; HRMS (ESI) : $\mathrm{m} / \mathrm{z}[\mathrm{M}+\mathrm{H}]^{+}$calcd for $\left[\mathrm{C}_{21} \mathrm{H}_{16} \mathrm{O}_{3} \mathrm{~F}\right]^{+}$: 335.1078; found : 335.1074 .

Methyl 4-(2-hydroxy-2-phenylethyl)benzoate (5a). Following the general procedure B with methyl 4-(bromomethyl)benzoate and benzaldehyde, 5a was purified by PE/EtOAc (from 90/10 to 80/20). $26.7 \mathrm{mg}$, yield: $52 \%$; yellow solid; mp $75-77{ }^{\circ} \mathrm{C}$; ${ }^{1} \mathrm{H}$ NMR $(250 \mathrm{MHz}$, $\left.\mathrm{CDCl}_{3}\right) \delta 7.99(\mathrm{~d}, J=8.1 \mathrm{~Hz}, 2 \mathrm{H}), 7.38-7.29(\mathrm{~m}, 5 \mathrm{H}), 7.25(\mathrm{~d}, J=8.1$ $\mathrm{Hz}, 2 \mathrm{H}), 4.96(\mathrm{~m}, 1 \mathrm{H}), 3.94(\mathrm{~s}, 3 \mathrm{H}), 3.17-3.06(\mathrm{~m}, 2 \mathrm{H}), 2.15$ (br-s, $1 \mathrm{H})$. Spectral data match those previously reported. ${ }^{16}$

Methyl 4-(2-(4-fluorophenyl)-2-hydroxyethyl)benzoate

$(5 b)$. Following the general procedure $B$ with methyl 4(bromomethyl)benzoate and 4-fluorobenzaldehyde, 5b was purified by PE/EtOAc (from $90 / 10$ to $80 / 20$ ). $35.0 \mathrm{mg}$, yield: $64 \%$; white solid; mp $114-116{ }^{\circ} \mathrm{C} ;{ }^{1} \mathrm{H}$ NMR $\left(250 \mathrm{MHz}, \mathrm{CDCl}_{3}\right) \delta 7.94(\mathrm{~d}, J=8.2$ $\mathrm{Hz}, 2 \mathrm{H}), 7.30-7.19(\mathrm{~m}, 4 \mathrm{H}), 7.07-6.97(\mathrm{~m}, 2 \mathrm{H}), 4.94-4.89(\mathrm{~m}, 1 \mathrm{H})$, $3.90(\mathrm{~s}, 6 \mathrm{H}), 3.08-3.04(\mathrm{~m}, 2 \mathrm{H}), 2.01(\mathrm{~s}, 1 \mathrm{H})$. Spectral data match those previously reported. ${ }^{17}$

4-(2-(4-fluorophenyl)-2-hydroxyethyl)-N,N-dimethylbenzamide (5c). Following the general procedure $\mathrm{B}$ with 4-(chloromethyl)- $\mathrm{N}, \mathrm{N}$ dimethylbenzamide and 4-fluorobenzaldehyde (10 equiv.), 5c was purified by PE/EtOAc (from 50/50 to 30/70). $24.2 \mathrm{mg}$, yield: $42 \%$; white solid; mp 96-98 ${ }^{\circ} \mathrm{C} ;{ }^{1} \mathrm{H}$ NMR $\left(400 \mathrm{MHz}, \mathrm{CDCl}_{3}\right) \delta 7.34(\mathrm{~d}, J=$ $8.1 \mathrm{~Hz}, 2 \mathrm{H}), 7.31-7.27(\mathrm{~m}, 2 \mathrm{H}), 7.18(\mathrm{~d}, J=8.1 \mathrm{~Hz}, 2 \mathrm{H}), 7.03-6.99$ $(\mathrm{m}, 2 \mathrm{H}), 4.90-4.86(\mathrm{~m}, 1 \mathrm{H}), 3.15-2.95(\mathrm{~m}, 8 \mathrm{H}), 1.87(b r-\mathrm{s}, 1 \mathrm{H})$; ${ }^{13} \mathrm{C}\left\{{ }^{1} \mathrm{H}\right\}$ NMR $\left(101 \mathrm{MHz}, \mathrm{CDCl}_{3}\right) \delta 171.7,163.6\left(\mathrm{C}-\mathrm{F},{ }^{1} \mathrm{JC}-\mathrm{F}=246.4\right.$ $\mathrm{Hz}), 161.2\left(\mathrm{C}-\mathrm{F},{ }^{1} \mathrm{JC}-\mathrm{F}=246.4 \mathrm{~Hz}\right), 139.6,139.49\left(\mathrm{C}-\mathrm{F},{ }^{4} \mathrm{JC}-\mathrm{F}=3.0\right.$ $\mathrm{Hz}), 139.46\left(\mathrm{C}-\mathrm{F},{ }^{4} \mathrm{JC}-\mathrm{F}=3.0 \mathrm{~Hz}\right), 134.7,129.6,127.74\left(\mathrm{C}-\mathrm{F},{ }^{3} \mathrm{JC}-\mathrm{F}=\right.$ $8.1 \mathrm{~Hz}), 127.66\left(\mathrm{C}-\mathrm{F},{ }^{3} \mathrm{JC}-\mathrm{F}=8.1 \mathrm{~Hz}\right), 127.5,115.5\left(\mathrm{C}-\mathrm{F},{ }^{2} \mathrm{JC}-\mathrm{F}=\right.$ $21.2 \mathrm{~Hz}), 115.3\left(\mathrm{C}-\mathrm{F},{ }^{2} \mathrm{JC}-\mathrm{F}=21.2 \mathrm{~Hz}\right), 74.7,46.0,39.7,35.5$; HRMS (ESI) : $\mathrm{m} / z[\mathrm{M}+\mathrm{Na}]^{+}$calcd for $\left[\mathrm{C}_{17} \mathrm{H}_{18} \mathrm{NO}_{2} \mathrm{FNa}\right]^{+}: 310.1214$; found : 310.1208 .

1-(4-fluorophenyl)-2-(4-vinylphenyl)ethanol (5d). Following the general procedure A with 4-vinylbenzyl chloride and 4fluorobenzaldehyde, 5d was purified by $\mathrm{PE} / \mathrm{EtOAc}$ (from $95 / 5$ to 85/15). $20.7 \mathrm{mg}$, yield: $43 \%$; yellow solid; mp 59-61 ${ }^{\circ} \mathrm{C}$; ${ }^{1} \mathrm{H}$ NMR $\left(400 \mathrm{MHz}, \mathrm{CDCl}_{3}\right) \delta 7.35-7.29(\mathrm{~m}, 4 \mathrm{H}), 7.12(\mathrm{~d}, J=8.1 \mathrm{~Hz}, 2 \mathrm{H})$, $7.05-7.00(\mathrm{~m}, 2 \mathrm{H}), 6.70(\mathrm{dd}, J=17.6,10.9 \mathrm{~Hz}, 1 \mathrm{H}), 5.72(\mathrm{dd}, J=$ $17.6,0.9 \mathrm{~Hz}, 1 \mathrm{H}), 5.23(\mathrm{dd}, J=10.9,0.8 \mathrm{~Hz}, 1 \mathrm{H}), 4.88(\mathrm{dd}, J=7.7$, $5.6 \mathrm{~Hz}, 1 \mathrm{H}), 3.00-2.97(\mathrm{~m}, 2 \mathrm{H}), 1.95(b r-\mathrm{s}, 1 \mathrm{H}) ;{ }^{13} \mathrm{C}\left\{{ }^{1} \mathrm{H}\right\}$ NMR $(101$ $\left.\mathrm{MHz}^{\mathrm{CDCl}}{ }_{3}\right) \delta 163.6\left(\mathrm{C}-\mathrm{F},{ }^{1} \mathrm{JC}-\mathrm{F}=246.4 \mathrm{~Hz}\right), 161.1\left(\mathrm{C}-\mathrm{F},{ }^{1} \mathrm{JC}-\mathrm{F}=\right.$ $246.4 \mathrm{~Hz}), 139.59\left(\mathrm{C}-\mathrm{F},{ }^{4} \mathrm{JC}-\mathrm{F}=3.0 \mathrm{~Hz}\right), 139.56\left(\mathrm{C}-\mathrm{F},{ }^{4} \mathrm{JC}-\mathrm{F}=3.0\right.$ $\mathrm{Hz}), 137.4,136.6,136.3,129.8,127.74\left(\mathrm{C}-\mathrm{F},{ }^{3} \mathrm{JC}-\mathrm{F}=8.1 \mathrm{~Hz}\right), 127.66$ $\left(\mathrm{C}-\mathrm{F},{ }^{3} \mathrm{JC}-\mathrm{F}=8.1 \mathrm{~Hz}\right), 126.5,115.5\left(\mathrm{C}-\mathrm{F},{ }^{2} \mathrm{JC}-\mathrm{F}=22.2 \mathrm{~Hz}\right), 115.3(\mathrm{C}-$ $\left.\mathrm{F},{ }^{2} \mathrm{JC}-\mathrm{F}=22.2 \mathrm{~Hz}\right), 113.7,74.8,46.0$; HRMS (ESI) $\left.: \mathrm{m} / z . \mathrm{M}+\mathrm{Na}\right]^{+}$ calcd for $\left[\mathrm{C}_{16} \mathrm{H}_{15} \mathrm{OFNa}\right]^{+}: 265.0999$; found : 265.0996 .

2-(2-chlorophenyl)-1-(2-fluorophenyl)ethanol (5e). Following the general procedure A with 2-chlorobenzyl chloride and 2fluorobenzaldehyde (10 equiv.), 5e was purified by $\mathrm{PE} / \mathrm{EtOAc}$ (90/10). $15.1 \mathrm{mg}$, yield: $30 \%$; white solid; mp $48-50{ }^{\circ} \mathrm{C} ;{ }^{1} \mathrm{H}$ NMR $\left(400 \mathrm{MHz}, \mathrm{CDCl}_{3}\right) \delta 7.50(\mathrm{td}, J=7.6,1.6 \mathrm{~Hz}, 1 \mathrm{H}), 7.42-7.34(\mathrm{~m}$, $1 \mathrm{H}), 7.30-7.25(\mathrm{~m}, 1 \mathrm{H}), 7.24-7.14(\mathrm{~m}, 4 \mathrm{H}), 7.05-7.00(\mathrm{~m}, 1 \mathrm{H}), 5.34$ 
$(\mathrm{dd}, J=8.7,4.5 \mathrm{~Hz}, 1 \mathrm{H}), 3.27(\mathrm{dd}, J=13.7,4.5 \mathrm{~Hz}, 1 \mathrm{H}), 3.14(\mathrm{dd}, J$ $=13.7,8.8 \mathrm{~Hz}, 1 \mathrm{H}), 1.63(b r-\mathrm{s}, 1 \mathrm{H}) ;{ }^{13} \mathrm{C}\left\{{ }^{1} \mathrm{H}\right\}$ NMR $(101 \mathrm{MHz}$, $\left.\mathrm{CDCl}_{3}\right) \delta 161.2\left(\mathrm{C}-\mathrm{F},{ }^{1} \mathrm{JC}-\mathrm{F}=247.5 \mathrm{~Hz}\right), 158.8\left(\mathrm{C}-\mathrm{F},{ }^{1} \mathrm{JC}-\mathrm{F}=247.5\right.$ $\mathrm{Hz}), 135.7,134.7,132.1,130.9\left(\mathrm{C}-\mathrm{F},{ }^{2} \mathrm{JC}-\mathrm{F}=13.1 \mathrm{~Hz}\right), 130.8(\mathrm{C}-\mathrm{F}$, $\left.{ }^{2} \mathrm{JC}-\mathrm{F}=13.1 \mathrm{~Hz}\right), 129.8,129.25\left(\mathrm{C}-\mathrm{F},{ }^{3} \mathrm{JC}-\mathrm{F}=8.1 \mathrm{~Hz}\right), 129.17(\mathrm{C}-\mathrm{F}$, $\left.{ }^{3} \mathrm{JC}-\mathrm{F}=8.1 \mathrm{~Hz}\right), 128.3,127.52\left(\mathrm{C}-\mathrm{F},{ }^{3} \mathrm{JC}-\mathrm{F}=4.5 \mathrm{~Hz}\right), 127.48(\mathrm{C}-\mathrm{F}$, $\left.{ }^{3} \mathrm{JC}-\mathrm{F}=4.5 \mathrm{~Hz}\right), 126.9,124.45\left(\mathrm{C}-\mathrm{F},{ }^{4} \mathrm{JC}-\mathrm{F}=3.5 \mathrm{~Hz}\right), 124.41(\mathrm{C}-\mathrm{F}$, $\left.{ }^{4} \mathrm{JC}-\mathrm{F}=3.5 \mathrm{~Hz}\right), 115.6\left(\mathrm{C}-\mathrm{F},{ }^{2} \mathrm{JC}-\mathrm{F}=22.2 \mathrm{~Hz}\right), 115.4\left(\mathrm{C}-\mathrm{F},{ }^{2} \mathrm{JC}-\mathrm{F}=\right.$ $22.2 \mathrm{~Hz}), 68.18\left(\mathrm{C}-\mathrm{F},{ }^{3} \mathrm{JC}-\mathrm{F}=2.1 \mathrm{~Hz}\right), 68.15\left(\mathrm{C}-\mathrm{F},{ }^{3} \mathrm{JC}-\mathrm{F}=2.1 \mathrm{~Hz}\right)$, 42.3; HRMS (ESI) : $\mathrm{m} / \mathrm{z}[\mathrm{M}+\mathrm{Na}]^{+}$calcd for $\left[\mathrm{C}_{14} \mathrm{H}_{12} \mathrm{OFClNa}\right]^{+}$: 273.0453; found : 273.0452 .

1-(4-fluorophenyl)-2-(2-iodophenyl)ethanol (5f). Following the general procedure $B$ with 2-iodobenzyl bromide and 4fluorobenzaldehyde (10 equiv.), 5f was purified by $\mathrm{PE} / \mathrm{EtOAc}$ (90/10). $16.8 \mathrm{mg}$, yield: $25 \%$; white solid; mp $69-71{ }^{\circ} \mathrm{C} ;{ }^{1} \mathrm{H}$ NMR $\left(400 \mathrm{MHz}, \mathrm{CDCl}_{3}\right) \delta 7.86(\mathrm{dd}, J=7.9,1.1 \mathrm{~Hz}, 1 \mathrm{H}), 7.41-7.37(\mathrm{~m}$, 2H), 7.29-7.25 (m, 1H), 7.16 (dd, $J=7.6,1.7 \mathrm{~Hz}, 1 \mathrm{H}), 7.06-7.02(\mathrm{~m}$, $2 \mathrm{H}), 6.94(\mathrm{td}, J=7.7,1.7 \mathrm{~Hz}, 1 \mathrm{H}), 5.01(\mathrm{dd}, J=8.4,4.9 \mathrm{~Hz}, 1 \mathrm{H})$, 3.16-3.05 (m, 2H), $1.60(b r-s, 1 \mathrm{H}) ;{ }^{13} \mathrm{C}\left\{{ }^{1} \mathrm{H}\right\}$ NMR $\left(101 \mathrm{MHz}, \mathrm{CDCl}_{3}\right)$ $\delta 163.7\left(\mathrm{C}-\mathrm{F},{ }^{1} \mathrm{JC}-\mathrm{F}=246.4 \mathrm{~Hz}\right), 161.2\left(\mathrm{C}-\mathrm{F},{ }^{1} \mathrm{JC}-\mathrm{F}=246.4 \mathrm{~Hz}\right)$, 140.7, 139.9, $139.56\left(\mathrm{C}-\mathrm{F},{ }^{4} \mathrm{JC}-\mathrm{F}=3.0 \mathrm{~Hz}\right), 139.53\left(\mathrm{C}-\mathrm{F},{ }^{4} \mathrm{JC}-\mathrm{F}=3.0\right.$ $\mathrm{Hz}), 131.4,128.8,128.4,127.6\left(\mathrm{C}-\mathrm{F},{ }^{3} \mathrm{JC}-\mathrm{F}=8.1 \mathrm{~Hz}\right), 127.5(\mathrm{C}-\mathrm{F}$, $\left.{ }^{3} \mathrm{JC}-\mathrm{F}=8.1 \mathrm{~Hz}\right), 115.5\left(\mathrm{C}-\mathrm{F},{ }^{2} \mathrm{JC}-\mathrm{F}=21.2 \mathrm{~Hz}\right), 115.3\left(\mathrm{C}-\mathrm{F},{ }^{2} \mathrm{JC}-\mathrm{F}=\right.$ $21.2 \mathrm{~Hz}), 101.2,73.1,50.7$; HRMS (ESI) : $\mathrm{m} / \mathrm{z}[\mathrm{M}+\mathrm{Na}]^{+}$calcd for $\left[\mathrm{C}_{14} \mathrm{H}_{12} \mathrm{OFINa}\right]^{+}$: 364.9809 ; found : 364.9808 .

Methyl 4-(1-(4-fluorophenyl)-1-hydroxypropan-2-yl)benzoate (5g). Following the general procedure $B$ with methyl 4-(1bromoethyl)benzoate and 4-fluorobenzaldehyde, $\mathbf{5 g}$ was purified by $\mathrm{PE} /$ EtOAc (from $95 / 5$ to $90 / 10$ ) as a $1: 1$ ratio of diastereoisomers. $29.9 \mathrm{mg}$, yield: $52 \%$; white solid; mp $157-159^{\circ} \mathrm{C} ;{ }^{1} \mathrm{H}$ NMR (400 $\mathrm{MHz}, \mathrm{CDCl}_{3}$ ) (mixture of diastereoisomers) $\delta 8.00(\mathrm{~d}, J=8.3 \mathrm{~Hz}$, $2 \mathrm{H}), 7.89(\mathrm{~d}, J=8.3 \mathrm{~Hz}, 2 \mathrm{H}), 7.32(\mathrm{~d}, J=8.3 \mathrm{~Hz}, 2 \mathrm{H}), 7.29-7.25(\mathrm{~m}$, $2 \mathrm{H}), 7.14(\mathrm{~d}, J=8.3 \mathrm{~Hz}, 2 \mathrm{H}), 7.12-7.08(\mathrm{~m}, 2 \mathrm{H}), 7.05-7.00(\mathrm{~m}, 2 \mathrm{H})$, $6.93-6.89(\mathrm{~m}, 2 \mathrm{H}), 4.76(\mathrm{~d}, J=6.5 \mathrm{~Hz}, 1 \mathrm{H}), 4.71(\mathrm{~d}, J=8.2 \mathrm{~Hz}, 1 \mathrm{H})$, $3.91(\mathrm{~s}, 3 \mathrm{H}), 3.89(\mathrm{~s}, 3 \mathrm{H}), 3.13-3.04(\mathrm{~m}, 2 \mathrm{H}), 1.79(b r-\mathrm{s}, 2 \mathrm{H}), 1.36(\mathrm{~d}$, $J=7.0 \mathrm{~Hz}, 3 \mathrm{H}), 1.11(\mathrm{~d}, J=7.1 \mathrm{~Hz}, 3 \mathrm{H}) .{ }^{13} \mathrm{C}\left\{{ }^{1} \mathrm{H}\right\} \mathrm{NMR}(101 \mathrm{MHz}$, $\mathrm{CDCl}_{3}$ ) (mixture of diastereoisomers) $\delta 167.17,167.14,163.8(\mathrm{C}-\mathrm{F}$, $\left.{ }^{1} \mathrm{JC}-\mathrm{F}=246.4 \mathrm{~Hz}\right), 163.4\left(\mathrm{C}-\mathrm{F},{ }^{1} \mathrm{JC}-\mathrm{F}=246.4 \mathrm{~Hz}\right), 161.3\left(\mathrm{C}-\mathrm{F},{ }^{1} \mathrm{JC}-\mathrm{F}\right.$ $=246.4 \mathrm{~Hz}), 160.1\left(\mathrm{C}-\mathrm{F},{ }^{1} \mathrm{JC}-\mathrm{F}=246.4 \mathrm{~Hz}\right), 148.8,138.43\left(\mathrm{C}-\mathrm{F},{ }^{4} \mathrm{JC}-\right.$ $\mathrm{F}=3.0 \mathrm{~Hz}), 138.40\left(\mathrm{C}-\mathrm{F},{ }^{4} \mathrm{JC}-\mathrm{F}=3.0 \mathrm{~Hz}\right), 138.22\left(\mathrm{C}-\mathrm{F},{ }^{4} \mathrm{JC}-\mathrm{F}=3.0\right.$ $\mathrm{Hz}), 138.19$ (C-F, $\left.{ }^{4} \mathrm{JC}-\mathrm{F}=3.0 \mathrm{~Hz}\right), 130.0,129.6,128.9,128.57$ (C-F, $\left.{ }^{3} \mathrm{JC}-\mathrm{F}=8.1 \mathrm{~Hz}\right), 128.54,128.49\left(\mathrm{C}-\mathrm{F},{ }^{3} \mathrm{JC}-\mathrm{F}=8.1 \mathrm{~Hz}\right), 128.3,128.09$ $\left(\mathrm{C}-\mathrm{F},{ }^{3} \mathrm{JC}-\mathrm{F}=7.1 \mathrm{~Hz}\right), 128.02\left(\mathrm{C}-\mathrm{F},{ }^{3} \mathrm{JC}-\mathrm{F}=7.1 \mathrm{~Hz}\right), 115.5\left(\mathrm{C}-\mathrm{F},{ }^{2} \mathrm{JC}-\right.$ $\mathrm{F}=21.2 \mathrm{~Hz}), 115.24\left(\mathrm{C}-\mathrm{F},{ }^{2} \mathrm{JC}-\mathrm{F}=21.2 \mathrm{~Hz}\right), 115.15\left(\mathrm{C}-\mathrm{F},{ }^{2} \mathrm{JC}-\mathrm{F}=\right.$ $22.2 \mathrm{~Hz}), 114.9\left(\mathrm{C}-\mathrm{F},{ }^{2} \mathrm{JC}-\mathrm{F}=22.2 \mathrm{~Hz}\right), 78.8,78.3,52.22,52.17$, $48.2,47.7,18.1,15.8$ (two aromatic carbons are missing due to overlap); HRMS (ESI) : $\mathrm{m} / z,[\mathrm{M}+\mathrm{Na}]^{+}$calcd for $\left[\mathrm{C}_{17} \mathrm{H}_{17} \mathrm{O}_{3} \mathrm{FNa}\right]^{+}$: 311.1054; found : 311.1052 .

Larger scale synthesis of $\mathbf{3 d}$. Following the general procedure A with 2-(chloromethyl)benzoate $\quad(155 \mu \mathrm{L}, \quad 1 \quad \mathrm{mmol}), \quad 2$ fluorobenzaldehyde $(316 \mu \mathrm{L}, 3 \mathrm{mmol})$ and SED-2 (427 mg, 1.5 $\mathrm{mmol}$ ) in DMF ( $5 \mathrm{~mL}), 3 d$ was purified by PE/EtOAc (90/10). 167 $\mathrm{mg}$, yield: $69 \%$.

\section{ASSOCIATED CONTENT}

\section{Supporting Information}

The Supporting Information is available free of charge on the ACS Publications website.

${ }^{1} \mathrm{H}$ NMR spectra for known compounds and ${ }^{1} \mathrm{H}$ and ${ }^{13} \mathrm{C}$ NMR spectra for new compounds (PDF)

\section{AUTHOR INFORMATION}

\section{Corresponding Author}

*E-mail: cedric.spitz@univ-amu.fr
*E-mail: patrice.vanelle@univ-amu.fr

\section{ORCID}

Cédric Spitz: 0000-0002-6473-3073

\section{Author Contributions}

All authors have given approval to the final version of the manuscript.

\section{Notes}

The authors declare no competing financial interest.

\section{ACKNOWLEDGMENT}

This work was supported by the CNRS and Aix-Marseille University. The authors thank the Spectropole team for elemental analysis. We express our thanks to V. Remusat for recording ${ }^{1} \mathrm{H}$ NMR and ${ }^{13} \mathrm{C}$ NMR spectra.

\section{REFERENCES}

(1) (a) Kongsaeree, P.; Prabpai, S.; Sriubolmas, N.; Vongvein, C.; Wiyakrutta, S. Antimalarial Dihydroisocoumarins Produced by Geotrichum sp., an Endophytic Fungus of Crassocephalum crepidioides. J. Nat. Prod. 2003, 66, 709-711. (b) Chen, J.; Zhou, L.; Tan, C. K.; Yeung, Y-Y. An Enantioselective Approach toward 3,4Dihydroisocoumarin through the Bromocyclization of Styrene-type Carboxylic Acids. J. Org. Chem 2012, 77, 999-1009. (c) Guimarães, K. G.; de Freitas, R. P.; Ruiz, A. L. T. G.; Fiorito, G. F.; de Carvalho, J. E.; da Cunha, E. F. F.; Ramalho, T. C.; Alves, R. B. Synthesis, antiproliferative activities, and computational evaluation of novel isocoumarin and 3,4-dihydroisocoumarin derivatives. Eur. J. Med. Chem. 2016, 111, 103-113.

(2) (a) Baur, J. A.; Sinclair, D. A. Therapeutic potential of resveratrol: the in vivo evidence. Nat. Rev. Drug Discovery 2006, 5, 493-506. (b) Cirla, A.; Mann, J. Combretastatins: from natural products to drug discovery. Nat. Prod. Rep. 2003, 20, 558-564.

(3) (a) Chenxiao, Q.; Yang, S.; Gandon, V.; Leboeuf, D. Calcium(II)- and Triflimide-Catalyzed Intramolecular Hydroacyloxylation of Unactivated Alkenes in Hexafluoroisopropanol. Org. Lett. 2019, 21, 7405-7409. (b) Nguyen, T. T.; Grigorjeva, L.; Daugulis, O. Cobalt-Catalyzed Coupling of Benzoic Acid C-H Bonds with Alkynes, Styrenes, and 1,3-Dienes. Angew. Chem. Int. Ed. 2018, 57, 1688-1691. (c) Organometallics in Synthesis-A Manual, 2nd ed.; Schlosser, M., Ed.; Wiley: Chichester, U.K., 2004. (d) Khoumeri, O.; Terme, T.; Vanelle, P. First TDAE Reactivity Using Benzonitrile Derivatives as Substrates and Its Application to the Synthesis of 3-Substituted Isochroman-1ones. Synthesis 2018, 50, 2617-2623.

(4) Duhamel, T.; Muniz, K. Cooperative iodine and photoredox catalysis for direct oxidative lactonization of carboxylic acids. Chem. Commun., 2019, 55, 933-936.

(5) (a) Giuglio-Tonolo, G.; Terme, T.; Médebielle, M.; Vanelle, P. Original reaction of $p$-nitrobenzyl chloride with aldehydes using tetrakis(dimethylamino)ethylene (TDAE). Tetrahedron Lett. 2003, 44, 6433-6435. (b) Giuglio-Tonolo, G.; Terme, T.; Médebielle, M.; Vanelle, P. Nitrobenzylation of $\alpha$-carbonyl ester derivatives using TDAE approach. Tetrahedron Lett. 2004, 45, 5121-5124. (c) AmiriAttou, O.; Terme, T.; Vanelle, P. Functionalization of 6Nitrobenzo[1,3]dioxole with Carbonyl Compounds via TDAE methodology Molecules 2005, 10, 545-551. (d) Montana, M.; Terme, T.; Vanelle, P. Original synthesis of $\alpha$-chloroketones in azaheterocyclic series using TDAE approach Tetrahedron Lett. 2006, 47, 6573-6576. (e) Khoumeri, O.; Terme, T.; Vanelle, P. Original and efficient synthesis of substituted 3,4-Dihydronaphtho[2,3-g]quinoline- 
2,6,11(1H)-triones. Synthesis 2009, 3677-3683. (f) Spitz, C.; Khoumeri, O.; Terme, T.; Vanelle, P. Diastereoselective Synthesis of $N$-tert-Butanesulfinylamines through Addition of $p$-Nitrobenzyl Chloride to $N$-tert-Butanesulfinimines Using a TDAE Strategy. Synlett 2013, 24, 1725-1727. (g) Spitz, C.; Lin, A.; Terme, T.; Vanelle, P. Mild and Metal-Free Diastereoselective Synthesis of $N$ tert-Butanesulfinylamines by Using Tetrakis(dimethylamino)ethylene. Synthesis 2014, 46, 3229-3232.

(6) (a) Murphy, J. A.; Khan, T. A.; Zhou, S.-Z.; Thomson, D. W.; Mahesh, M. Highly Efficient Reduction of Unactivated Aryl and Alkyl Iodides by a Ground-State Neutral Organic Electron Donor. Angew. Chem. Int. Ed. 2005, 44, 1356-1360. (b) Broggi, J.; Terme, T.; Vanelle, P. Organic Electron Donors as Powerful Single-Electron Reducing Agents in Organic Synthesis. Angew. Chem. Int. Ed. 2014, 53, 384-413. (c) Doni, E.; Murphy, J. A. Evolution of neutral organic super-electrondonors and their applications. Chem. Commun. 2014, 50, 6073-6087. (d) Murphy, J. A. Discovery and Development of Organic Super-Electron-Donors. J. Org. Chem 2014, 79, 3731-3746.

(7) (a) Hanson, S. S.; Doni, E.; Traboulsee, K. T.; Coulthard, G.; Murphy, J. A.; Dyker, C. A. Pushing the Limits of Neutral Organic Electron Donors: A Tetra(iminophosphorano)-Substituted Bispyridinylidene. Angew. Chem. Int. Ed. 2015, 54, 11236-11239. (b) Hanson, S. S.; Richard, N. A.; Dyker, C. A. Powerful Bispyridinylidene Organic Reducing Agents with Iminophosphorano $\pi$-Donor Substituents. Chem. Eur. J. 2015, 21, 8052-8055. (c) Burgoyne, M. M.; MacDougall, T. M.; Haines, Z. N.; Conrad, J. W.; Calhoun, L. A.; Decken, A.; Dyker, C. A. A strong organic electron donor incorporating highly $\pi$-donating triphenylphosphonium ylidyl substituents. Org. Biomol. Chem., 2019, 17, 9726-9733.

(8) Glaser, F.; Larsen, C. B.; Kerzig, C.; Wenger ; O. S. Aryl dechlorination and defluorination with an organic superphotoreductant. Photochem. Photobiol. Sci. 2020, 19, 1035-1041.

(9) (a) Schoenebeck, F.; Murphy, J. A.; Zhou, S.-Z.; Uenoyama, Y.; Miclo, Y.; Tuttle, T. Reductive Cleavage of Sulfones and Sulfonamides by a Neutral Organic Super-Electron-Donor (S.E.D.)
Reagent. J. Am. Chem. Soc. 2007, 129, 44, 13368-13369. (b) Jolly, P. I.; Fleary-Roberts, N.; O'Sullivan, S.; Doni, E.; Zhou, S.-Z.; Murphy, J. A. Reactions of triflate esters and triflamides with an organic neutral super-electron-donor. Org. Biomol. Chem. 2012, 10, 58075810 .

(10) Smith, A. J.; Poole, D. L.; Murphy, J. A. The role of organic electron donors in the initiation of BHAS base-induced coupling reactions between haloarenes and arenes. Sci. China Chem. 2019, 62, $1425-1438$.

(11) Murphy, J. A.; Garnier, J.; Park, S. R.; Schoenebeck, F.; Zhou, S.-Z; Turner, A. T. Super-Electron Donors: Bis-pyridinylidene Formation by Base Treatment of Pyridinium Salts. Org. Lett. 2008, $10,1227-1230$

(12) Wilsey, S.; Dowd, P.; Houk, K. N. Effect of Alkyl Substituents and Ring Size on Alkoxy Radical Cleavage Reactions. J. Org. Chem. 1999, 64, 8801-8811.

(13) Mandal, S. K.; Roy, S. C. Titanocene(III) chloride mediated radical-induced synthesis of 3,4-dihydroisocoumarins: synthesis of ( \pm -hydrangenol, $( \pm)$-phyllodulcin, $( \pm)$-macrophyllol and $( \pm)$ thunberginol G. Tetrahedron 2008, 64, 11050-11057.

(14) Crespi, S.; Jäger, S.; König, B.; Fagnoni, M. A Photocatalytic Meerwein Approach to the Synthesis of Isochromanones and Isochromenones. Eur. J. Org. Chem. 2017, 2147-2153.

(15) Bellinger, G. C. A.; Campbell, W. E.; Giles, R. G. F.; Tobias, J. D. Formation of some 3-Aryl-3,4-dihydroisocoumarins by Thermal Ring Closure of Stilbene-2-carboxylic Acids J. Chem. Soc. Perkin Trans. 1 1982, 2819-2825.

(16) Wang, J.; Xue, L.; Hong, M.; Ni, B.; Niu, T. Heterogeneous visible-light-induced Meerwein hydration reaction of alkenes in water using mpg- $\mathrm{C}_{3} \mathrm{~N}_{4}$ as a recyclable photocatalyst Green Chem. 2020, 22, 411-416.

(17) Liao, L-L.; Cao, G-M.; Ye, J-H.; Sun, G-Q.; Zhou, W-J.; Gui, Y-Y.; Yan, S-S.; Shen, G.; Yu, D-G. Visible-Light-Driven ExternalReductant-Free Cross-Electrophile Couplings of Tetraalkyl Ammonium Salts J. Am. Chem. Soc. 2018, 140, 17338-17342. 